\title{
How intermittency affects the rate at which rainfall extremes respond to changes in temperature
}

\author{
Marc Schleiss \\ Department of Geoscience \& Remote Sensing, Delft University of Technology, Delft, the Netherlands
}

Correspondence: Marc Schleiss (m.a.schleiss@tudelft.nl)

Received: 10 January 2018 - Discussion started: 22 January 2018

Accepted: 13 June 2018 - Published: 9 July 2018

\begin{abstract}
A detailed analysis of how intermittency (i.e., the alternation of dry and rainy periods) modulates the rate at which sub-daily rainfall extremes depend on temperature is presented. Results show that hourly extremes tend to be predominantly controlled by peak intensity, increasing at a rate of approximately $7 \%$ per degree in agreement with the Clausius-Clapeyron equation. However, a rapid increase in intermittency upward of $20-25^{\circ} \mathrm{C}$ is shown to produce local deviations from this theoretical scaling, resulting in lower scaling rates. On the other hand, rapidly decreasing intermittency with temperature between 10 and $20^{\circ}$ can result in higher net scaling rates than expected, potentially exceeding Clausius-Clapeyron. In general, the importance of intermittency in controlling the scaling rates of precipitation with temperature grows as we progress from hourly to daily aggregation timescales and beyond. Thermodynamic effects still play an important role in controlling the maximum water-holding capacity of the atmosphere and therefore peak rainfall intensity, but the observational evidence shows that, beyond a few hours, storm totals become increasingly dominated by dynamical factors. The conclusion is that Clausius-Clapeyron scaling alone cannot be used to reliably predict the net effective changes in rainfall extremes with temperature beyond a few hours. A more general scaling model that takes into account simultaneous changes in intermittency and peak intensity with temperature is proposed to help better disentangle these two phenomena (e.g., peak intensity and intermittency). The new model is applied to a large number of high-resolution rain gauge time series in the United States, and results show that it greatly improves the representation of rainfall extremes with temperature, producing a much more consistent and reliable picture of extremes across scales than using Clausius-Clapeyron only.
\end{abstract}

\section{Introduction}

Recently, there has been an increased interest in understanding and predicting changes in precipitation extremes due to global warming (e.g., Trenberth et al., 2003; Frei et al., 2006; Allan and Soden, 2008; Trenberth, 2011; Muschinski and Katz, 2013; Westra et al., 2014; Ban et al., 2015; Groisman et al., 2015; Donat et al., 2016; Scherrer et al., 2016). Most studies on this topic agree that heavy rainfall is likely to increase in the future. However, a clear framework for predicting changes in rainfall intensities across scales is still lacking. The general consensus seems to be that in places with sufficient moisture availability, rainfall extremes will increase at the same rate as the moisture-holding capacity of the atmosphere, that is, at a rate of about $7 \%$ per degree of warming in accordance to the Clausius-Clapeyron relationship. The observational evidence, however, points to a more complicated picture (Shaw et al., 2011). It shows that rainfall extremes and temperatures are linked in more complicated ways, depending on the local climatology, moisture availability, large-scale forcing, orography and scale of analysis (e.g., Panthou et al., 2014; Donat et al., 2016; Drobinski et al., 2016; Ivancic and Shaw, 2016; Barbero et al., 2017). Some hourly rainfall extremes, for example, have been shown to increase at rates of up to $14 \%{ }^{\circ} \mathrm{C}^{-1}$ - about twice as fast as expected from Clausius-Clapeyron (Lenderink and van Meijgaard, 2008; Lenderink et al., 2011). Haerter and Berg (2009) and Berg et al. (2013) argue that this is due to fundamental differences in scaling between large-scale stratiform ex- 
tremes, which are expected to increase with temperature at the Clausius-Clapeyron rate, and small-scale convective extremes, which may exceed Clausius-Clapeyron due to dynamical processes like strong local updrafts and downdrafts. Lepore et al. (2015) argue along the same lines, highlighting the importance of separating the effects of temperature on rainfall extremes via increased atmospheric water content (described by Clausius-Clapeyron) and via enhanced atmospheric convection and moisture convergence.

One important yet poorly discussed issue in all these studies concerns the role played by intermittency (i.e., the alternation of dry and rainy periods) in controlling the response of rainfall extremes to changing temperatures. Indeed, beyond a few hours of aggregation timescale, total rainfall amounts often turn out to be more correlated to storm duration and intermittency rather than peak rainfall intensity (e.g., Azad and Sorteberg, 2017; Lamjiri et al., 2017). And while the discrete, episodic nature of precipitation may be most apparent at larger scales (e.g., days, weeks or months), its effects can be observed down to the microscale (e.g., Kumar and Foufoula-Georgiou, 1994; Ignaccolo et al., 2009; De Michele and Ignaccolo, 2013; Mascaro et al., 2013). An important yet still poorly documented issue in this context concerns the sensitivity of rainfall intermittency to changes in temperature across spatial and temporal aggregation scales. These variations have been shown to affect the rate at which extreme precipitation scales with temperature in ways that ClausiusClapeyron alone cannot explain (Haerter et al., 2010; Panthou et al., 2014; Wasko et al., 2015).

A recent study by Wasko et al. (2016) found that although peak rainfall intensity usually scales positively with temperature, event duration and spatial extent tend to decrease with temperature. Global climate model simulations by Dwyer and O'Gorman (2017) partly confirm this trend, projecting a global $1 \%$ decrease in extreme precipitation duration per degree of warming. However, the magnitude of this trend remains highly uncertain due to strong limitations in the ability of global climate models to simulate realistic intermittency patterns. A recently proposed idealized stochastic model by Neelin et al. (2017) gives additional insight into the main mechanisms responsible for controlling the complicated interaction between event duration, peak intensity and total rainfall amount. It shows that mathematically, the problem can be represented as a competition (over time) between moisture convergence and water losses by precipitation. While too idealized, the model still highlights some important aspects like the fact that unless there is rapid replenishment of moisture from surrounding regions (e.g., through advection), big storms with heavy precipitation at their core will tend to run out of moisture more quickly, forcing precipitation to cease earlier (Trenberth et al., 2003). The consequence of this is a more complicated scaling pattern in which rainfall extremes do not necessarily increase at the same rate for all temperatures and across all scales of aggregation. Temperature still plays a crucial role by limiting the maximum water-holding capacity of the air and controlling evaporation rates in surrounding regions. However, dynamical factors like intermittency also need to be considered.

The present study aims at shedding new light on this important issue by presenting a detailed statistical analysis of how intermittency modulates the rate at which precipitation extremes (in current climate) depend on temperature. Results show that at timescales of $1 \mathrm{~h}$ or less, rainfall extremes tend to be predominantly controlled by changes in temperature. However, rapid local increases or decreases in intermittency with temperature can significantly lower or amplify the net scaling rates. In extreme cases, this may lead to (locally) negative scaling rates or, conversely, super-Clausius-Clapeyron scaling. As we move towards rainfall extremes at daily scales and beyond, intermittency rapidly gains in importance, masking most of the thermodynamic effects. To disentangle the two, a more general scaling model that takes into account simultaneous changes in intermittency and maximum intensity with temperature is proposed. Results show the new model greatly improves predictions of rainfall extremes with temperature, producing a more consistent and reliable depiction of observed responses across a wide range of temporal scales.

The rest of this paper is structured as follows: Sect. 2 introduces the data used for the analysis. Section 3 describes the methods and models used to detect and analyze rainfall extremes. The main results are provided in Sect. 4. The first part focuses on how intermittency affects the scaling of rainfall extremes across temperatures and timescales. The second part analyzes the goodness of fit of the newly proposed model and the third and last part investigates the sensitivity of derived scaling rates with respect to the chosen quantile $q$. The conclusions and some additional ideas for future research are given in Sect. 5.

\section{Data}

The data used in the study were taken from the sub-hourly US Climate Reference Network (Diamond et al., 2013). Two main quantities were considered: total precipitation depth $R$ (expressed in $\mathrm{mm}$ ) and average air temperature $T$ (in Celsius) at constant $5 \mathrm{~min}$ temporal resolution from the beginning of January 2006 to the end of August 2017. Initial analyses also included dew point temperature $T_{d}$ (in Celsius), estimated by combining relative humidity and air temperature using the Magnus formula (e.g., Alduchov and Eskridge, 1996). However, relative humidity measurements started later and most of them were too short to derive reliable statistics. Therefore it was decided to focus solely on air temperature. Note that the $5 \mathrm{~min}$ temperature values were derived by averaging independent $10 \mathrm{~s}$ measurements from multiple colocated sensors. Similarly, precipitation totals (both solid and liquid) were estimated using a weighing bucket gauge with three independent load cell sensors. Automatic quality control was applied 


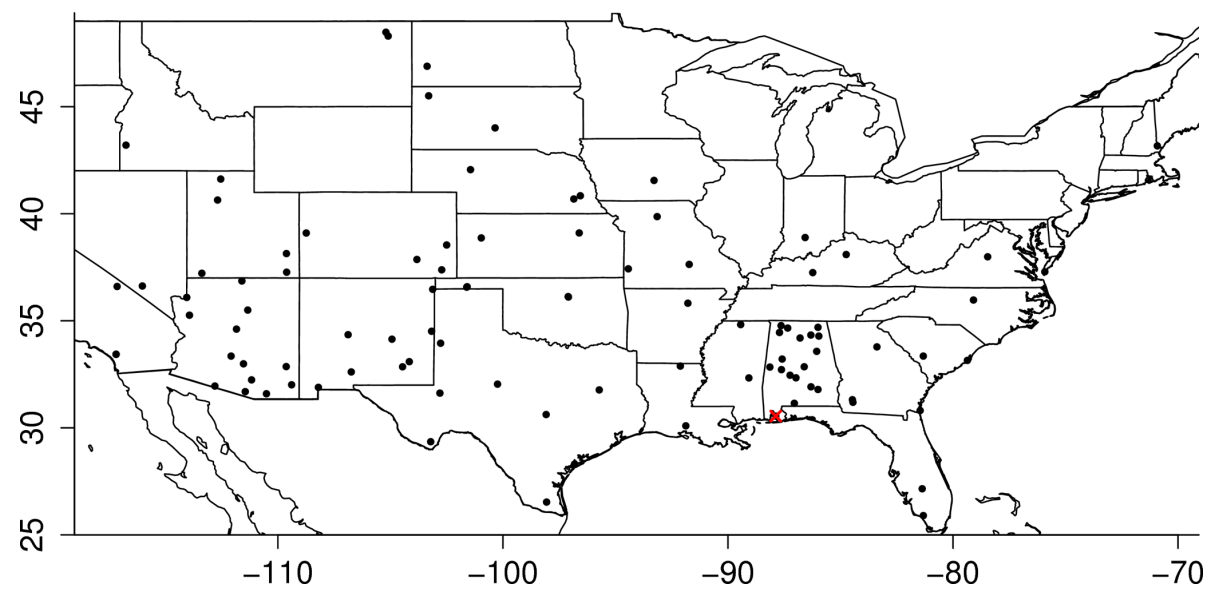

Figure 1. Map showing the location of the 99 selected USCRN stations. The red cross denotes station AL-Fairhope-3-NE, which was chosen for illustration purposes.

to the data, and all flagged measurements were removed prior to analysis.

Since the goal of this paper is to analyze the properties of rainfall extremes across different scales, all time series were aggregated from their original resolution of $5 \mathrm{~min}$ to larger timescales of 1,2 , etc., ... up to $24 \mathrm{~h}$ in regular steps of $1 \mathrm{~h}$. Aggregation was performed over overlapping time windows (shifted by $5 \mathrm{~min}$ ), taking the sums of all $5 \mathrm{~min}$ rainfall amounts in each time interval. Air temperature was aggregated using the arithmetic mean, and values were binned into regular classes of $1^{\circ} \mathrm{C}$. All aggregation time windows containing one or more missing values were discarded prior to analysis. The main reason for using overlapping time windows during aggregation was to better account for the fact that the starting time of an aggregation time period is arbitrary. By contrast, nonoverlapping time windows would have resulted in many large precipitation accumulations being missed.

The full weather station network consisted of 232 different stations spread across the US, Canada and Siberia. However, only a small subset of these stations were kept for the analysis. Specifically, only the time series with at least 20 valid positive rainfall values in at least 20 different temperature classes between 5 and $30^{\circ} \mathrm{C}$ at the $24 \mathrm{~h}$ aggregation timescale were kept. This drastically reduced the number of stations from 232 to 99 . A map with the 99 stations satisfying all these criteria is shown in Fig. 1. For illustration purposes, one randomly selected station (i.e., AL-Fairhope-3-NE) in the southern part of the country was selected (see red cross in Fig. 1). The station is representative of a humid subtropical climate with plenty of moisture availability and a mixture of both small and large-scale rainfall extremes.

\section{Methods}

\subsection{Definition of rainfall extremes}

Consider a time series of strictly positive rainfall amounts $R_{1}(\Delta t), \ldots, R_{N}(\Delta t)$ at temperatures $T_{1}(\Delta t), \ldots, T_{N}(\Delta t)$. Each $R_{i}(\Delta t)$ represents the total accumulated rainfall amount (in $\mathrm{mm}$ ) over a time period of length $\Delta t>0$. The couples $\left(R_{i}, T_{i}\right)$ can be seen as realizations of a bivariate random variable $\left(R_{\Delta t}, T_{\Delta t}\right)$ with a joint probability distribution function $F_{\Delta t}$. A rainfall amount $R_{i}(\Delta t)>0$ at timescale $\Delta t$ and temperature $T_{i}(\Delta t)=T$ is said to be "extreme" if it exceeds the $q$ th quantile $R_{q}(\Delta t, T)$ of all strictly positive rainfall amounts at this temperature and aggregation timescale:

$\mathbb{P}\left[R_{\Delta t} \leq R_{q}(\Delta t, T) \mid T_{\Delta t}=T\right]=q$,

where $\mathbb{P}$ denotes the probability and $0<q<1$ is the quantile of interest. To have enough observations, temperature measurements are binned in 20 different classes between 5 and $30^{\circ} \mathrm{C}$. Given the relatively small sample sizes (for each temperature class), the default quantile used in this paper is $q=0.95$. However, 50 other values of $q$ between 0.95 and 0.999 (in regular steps of 0.001) are also considered for the sensitivity analysis in the last part of the paper. To avoid large estimation errors, quantile $q>0.95$ was computed only if there were at least $\left\lceil\frac{1}{1-q}\right\rceil$ strictly positive rainfall observations in a given temperature class, with $\lceil x\rceil$ denoting the upper integer part of $x$. Consequently, not all 50 different quantiles are available for each of the 99 selected stations.

\subsection{Internal intermittency}

Consider an aggregated rainfall amount $R_{i}(\Delta t)$ at scale $\Delta t>0$ with $\Delta t=n \cdot \Delta t_{0}$. The variable $n \in \mathbb{N}$ represents the aggregation ratio with respect to a smaller observation scale $\Delta t_{0}$. For example, $\Delta t_{0}=5 \mathrm{~min}$ and $n=12$ for hourly 

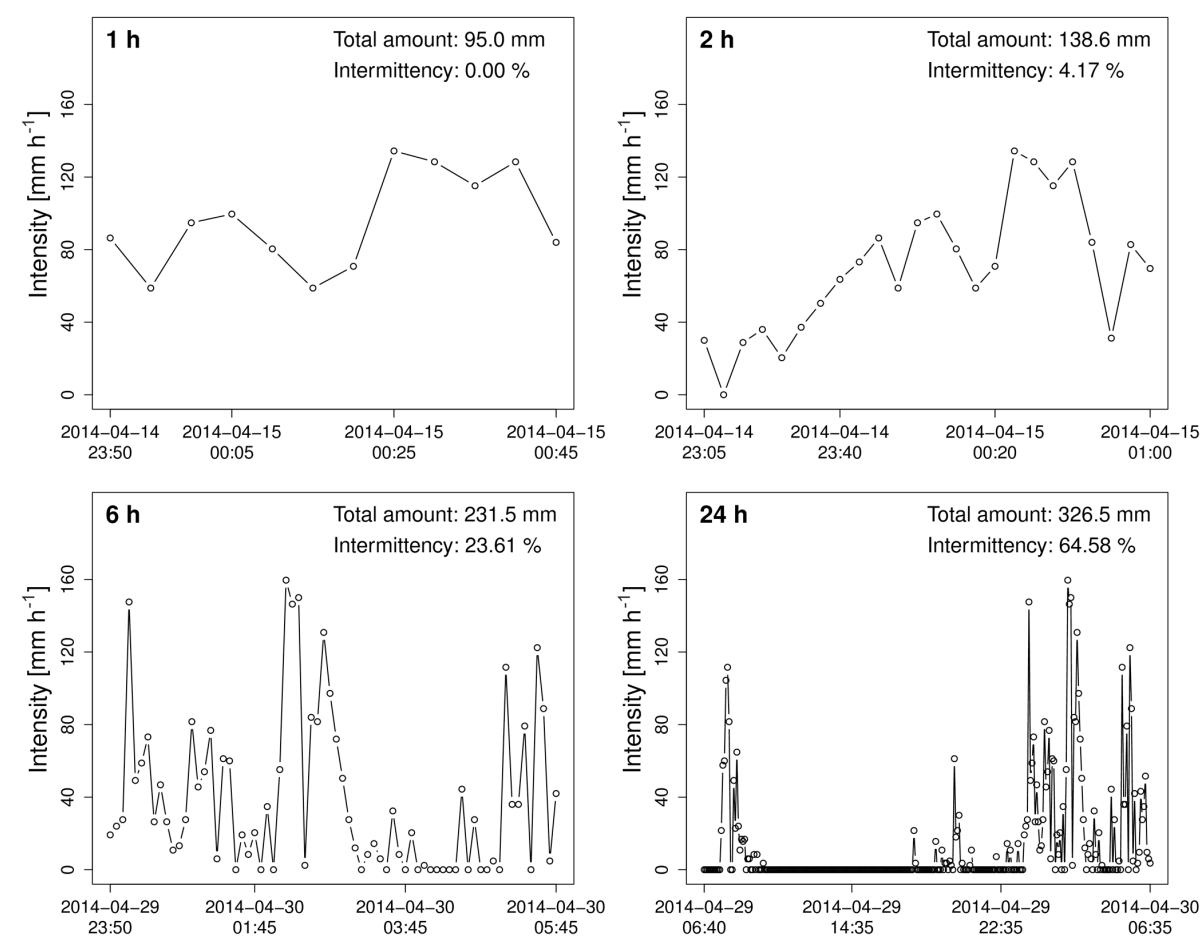

Figure 2. Highest rainfall accumulations recorded at AL-Fairhope-3-NE for 1, 2, 6 and $24 \mathrm{~h}$ aggregation timescales.

aggregated $5 \mathrm{~min}$ rainfall amounts and $n=288$ for daily aggregated 5 min amounts. By definition, each $R_{i}(\Delta t)$ can be expressed as the sum of $n$ individual observations at smaller scale $\Delta t_{0}$ :

$R_{i}\left(n \Delta t_{0}\right)=\sum_{j=n i-n+1}^{n i} R_{j}\left(\Delta t_{0}\right)$

The fraction of dry periods at reference scale $\Delta t_{0}$ contained within $\Delta t$ is called the internal intermittency of $R_{i}(\Delta t)$ and is denoted by

$$
I_{i}(\Delta t)=\frac{1}{n} \sum_{j=n i-n+1}^{n i} 1_{\left\{R_{j}\left(\Delta t_{0}\right)=0\right\}},
$$

where $1_{\{x\}}$ is a function that equals 1 if $x$ is true and 0 otherwise. Note that for more conciseness, the reference scale $\Delta t_{0}$ has been omitted in the notation for $I_{i}(\Delta t)$. However, it should be clear from the definition that $I_{i}(\Delta t)$ is a relative measure of lacunarity with respect to a fixed reference scale $\Delta t_{0}$, that is, a measure of the fraction of dry intervals of length $\Delta t_{0}$ contained within $\Delta_{t}$. The smaller the reference scale $\Delta t_{0}$, the larger the internal intermittency. Fortunately, this scale dependence is not a major problem here as we are mostly interested in understanding relative changes in intermittency from one temperature to another.

Also, note that because of the original sampling resolution of $5 \mathrm{~min}$ in the USCRN (US Climate Reference Network) data, the internal intermittency of a rainfall amount at scale $n \cdot \Delta t_{0}$ can only be estimated with an accuracy of at best $1 / n$. Small-scale intermittency estimates are therefore affected by relatively strong discretization effects that could potentially mask the changes from one temperature to another. To mitigate this effect, the smallest aggregation timescale considered in this paper will be $60 \mathrm{~min}(n=12)$, which means that the maximum uncertainty affecting intermittency estimates is $8.3 \%$. Fortunately, the uncertainty rapidly decreases with aggregation timescale to reach a minimum of $0.347 \%$ at the daily timescale.

\subsection{Intermittency of rainfall extremes}

The way rainfall intermittency varies with spatial and temporal aggregation scale has already been studied quite extensively (e.g., Kumar and Foufoula-Georgiou, 1994; Jeannin et al., 2008; Kundu and Siddani, 2011; Schleiss et al., 2011; Mascaro et al., 2013; Dunkerley, 2015). So far, however, very few studies have tried to characterize the conditional expectation of intermittency at a given rainfall intensity, temperature and scale. The latter plays a crucial role in many applications, including flood forecasting, radar remote sensing and the design of stochastic rainfall simulators capable of preserving the structure and dynamics of rainfall across scales.

Figure 2 shows the four largest observed rainfall accumulations at the 1,2,6 and $24 \mathrm{~h}$ aggregation timescales for the station in Fairhope, Alabama. As can be expected, rainfall amounts steadily increase with aggregation scale. Average rainfall intensities, on the other hand, decrease from 
$95 \mathrm{~mm} \mathrm{~h}^{-1}$ at the hourly timescale to less than $13.6 \mathrm{~mm} \mathrm{~h}^{-1}$ at the daily scale. A large part of this 7-fold decrease can be attributed to the strong increase in internal intermittency from roughly $0 \%$ at hourly timescale to more than $64 \%$ at the daily timescale, highlighting the fundamentally different physical processes through which extreme rainfall accumulations at small and large timescales are produced. In the following, we explain the methodology used in this paper to generalize this type of analysis to other rainfall quantiles and study variations in intermittency across scales and temperatures.

Similarly to rainfall and temperature, it is possible to represent the internal intermittency $I_{i}(\Delta t)$ of rainfall accumulations at a given timescale $\Delta t$ as realizations of a random variable $I_{\Delta t}$. Combining all the variables together produces triplets $\left(R_{\Delta t}, T_{\Delta t}, I_{\Delta t}\right)$ of simultaneous rainfall accumulations, temperatures and internal intermittency values. Detailed analysis of the joint and marginal distributions of this trivariate random function is necessary to fully understand the link between rainfall extremes and temperature across scales. Unfortunately, due to the short data record, this proves very challenging (especially for extremes). A simpler approach is to focus on the expected intermittency $I_{q}(\Delta t, T)$ of the $q$ th rainfall quantile $R_{q}(\Delta t, T)$ conditionally on temperature $T$ :

$I_{q}(\Delta t, T)=\mathbb{E}\left[I_{\Delta t} \mid R_{\Delta t}=R_{q}(\Delta t, T)\right.$ and $\left.T_{\Delta t}=T\right]$,

where $\mathbb{E}$ denotes the expectation operator. In this study, expected intermittency values (conditional on temperature) were estimated by fitting a logistic regression between the internal intermittency and the logit of $q$, as shown in Fig. 3:

$g\left(I_{q}(\Delta t, T)\right)=c_{0}(\Delta t, T)+c_{1}(\Delta t, T) g(q)$,

with the logit function $g(x)$ given by

$g(x)=\ln \left(\frac{x}{1-x}\right)$.

The two model parameters $c_{0}(\Delta t, T)$ and $c_{1}(\Delta t, T)$ are fitted numerically for each of the selected stations, temperature $T$ (between 5 and $30^{\circ} \mathrm{C}$ ) and aggregation timescale $\Delta t$ between 1 and $24 \mathrm{~h}$. The fit is performed using the $\operatorname{glm}$ () function in the statistical programming language $\mathrm{R}$ (R Core Team, 2017). The fitted model parameters $\hat{c}_{0}(\Delta t$, $T)$ and $\hat{c}_{1}(\Delta t, T)$ at each temperature $T$ can then be used to estimate the expected internal intermittency of extremes for any given rainfall quantile $q$ :

$\hat{I}_{q}(\Delta t, T)=g^{-1}\left(\hat{c}_{0}(\Delta t, T)+\hat{c}_{1}(\Delta t, T) g(q)\right)$,

with

$g^{-1}(x)=\frac{e^{x}}{e^{x}+1}$.
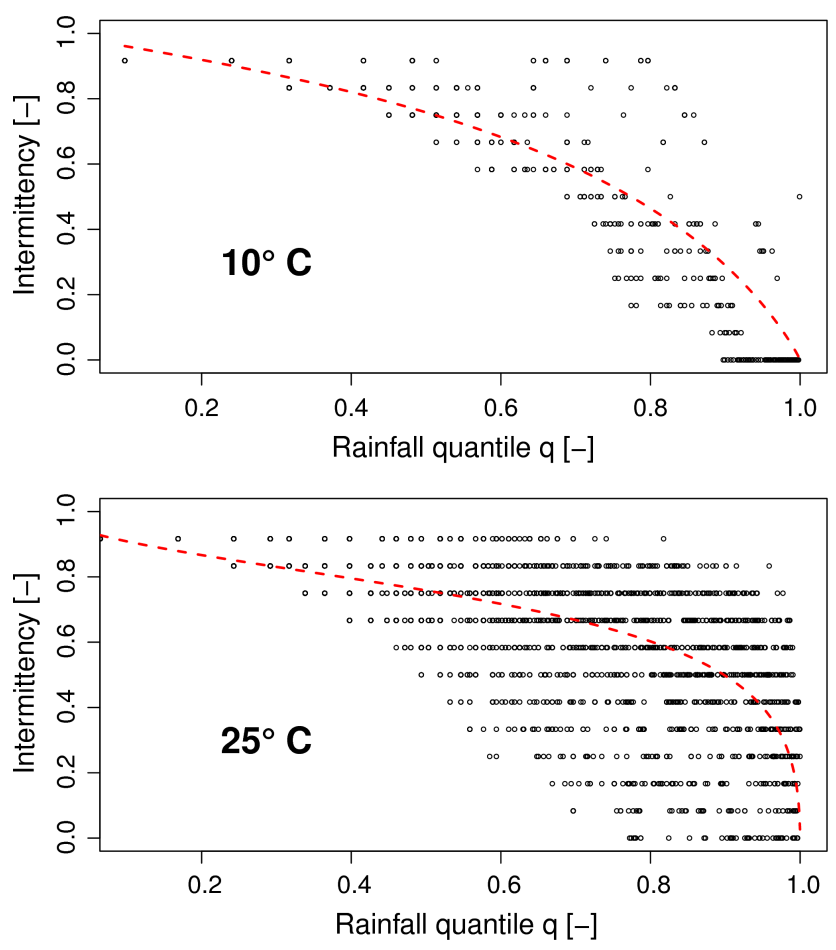

Figure 3. Logistic regression between intermittency and rainfall quantile at AL-Fairhope-3-NE for the $1 \mathrm{~h}$ timescale and two different temperatures $\left(10\right.$ and $\left.25^{\circ} \mathrm{C}\right)$.

\subsection{Scaling of extremes}

Scaling analyses in this paper are performed by considering the mean air temperature $T_{i}(\Delta t)$ over the same aggregation time period than the rainfall amounts $R_{i}(\Delta t)$. Some previous studies suggested to use temperatures recorded immediately before/after the rain event to avoid potential "contamination" by the rain itself. However, averaging temperatures before/after an event is questionable for at least two reasons. Firstly, the start/end of an event are often very subjective and highly dependent on the scale of analysis. And secondly, prior/posterior temperatures may not necessarily have the same representativity depending on when rain occurred and how long it lasted. For these reasons, only simultaneous temperature and rainfall measurements will be used.

Previous studies have shown that rainfall extremes $R_{q}(\Delta t, T)$ for large $q$ increase approximately exponentially with temperature $T$ :

$R_{q}(\Delta t, T) \approx e^{\alpha_{q}(\Delta t)+\beta_{q}(\Delta t) T}$,

where $\alpha_{q}(\Delta t) \in \mathbb{R}$ and $\beta_{q}(\Delta t) \in \mathbb{R}$ are two parameters depending on the quantile $q$ and aggregation scale $\Delta t$. The exponential relationship is (partly) justified by the fact that saturation water vapor pressure exponentially grows with temperature, meaning that under conditions of constant relative humidity, precipitable water should scale approximately similarly (Trenberth et al., 2003). Assuming model (Eq. 9) holds, 
the scaling rate $\lambda_{q}(\Delta t)$ per unit increase in temperature $T$ is given by

$\lambda_{q}(\Delta t, T)=\frac{R_{q}(\Delta t, T+1)}{R_{q}(\Delta t, T)}-1=e^{\beta_{q}(\Delta t)}-1$,

which is a constant and does not depend on $T$. One of the main problems with the constant scaling assumption above is that it only seems to hold in approximation and over a limited range of temperatures. In particular, departures from loglinearity have been reported at temperatures below 5-10 and above $20-25^{\circ} \mathrm{C}$ (e.g., Lenderink and van Meijgaard, 2008; Haerter et al., 2010). One way to account for these deviations and increase robustness is to take the log transform of rainfall quantiles and derive the slope parameter $\beta_{q}(\Delta t)$ in Eq. (9) using the nonparametric Theil-Sen estimator (Sen, 1968) instead of traditional least squares. Even so, average scaling rates derived using this technique may not be very representative of the actual changes in extreme rainfall amounts across the whole temperature range.

To address this limitation, another slightly more general scaling model is proposed in which a multiplicative correction term is added in Eq. (9) to account for possible changes in intermittency with scale and temperature:

$R_{q}(\Delta t, T) \approx\left[1-I_{q}(\Delta t, T)\right] \cdot e^{\alpha_{q}^{\text {new }}(\Delta t)+\beta_{q}^{\text {new }}(\Delta t) T}$,

where $I_{q}(\Delta t, T)$ is the expected internal intermittency of rainfall extremes exceeding the $q$ th quantile (conditionally on temperature $T)$ and $\alpha_{q}^{\text {new }}(\Delta t)$ and $\beta_{q}^{\text {new }}(\Delta t)$ are two new model parameters. The idea is similar to that proposed by Wasko et al. (2015) in which the authors related peak rainfall intensity to temperature with a correction for storm duration. However, the parametric form of their model was slightly different from the one proposed here. Also, the main covariate modulating the rainfall amounts in Eq. (11) is internal intermittency and not rainfall duration.

Since the internal intermittency $I_{q}(\Delta t, T)$ in Eq. (11) changes with temperature, relative rates of increase/decrease in rainfall amounts per unit change in temperature are not independent of $T$ anymore like in Eq. (10) but modulated by local changes in intermittency:

$\lambda_{q}^{\text {new }}(\Delta t, T)=\frac{1-I_{q}(\Delta t, T+1)}{1-I_{q}(\Delta t, T)} e^{\beta_{q}^{\text {new }}(\Delta t)}-1$.

As a result, rainfall amounts can either increase or decrease with temperature, leading to a better differentiation between the thermodynamic effects (i.e., increase in moisture-holding capacity with temperature) and dynamic effects caused by changes in intermittency with temperature. In fact, since Eq. (11) is equivalent to a renormalization of the rainfall amounts by a factor $1-I_{q}(\Delta t, T)$, we can retrieve the "true" underlying scaling rate with temperature after correction for intermittency:
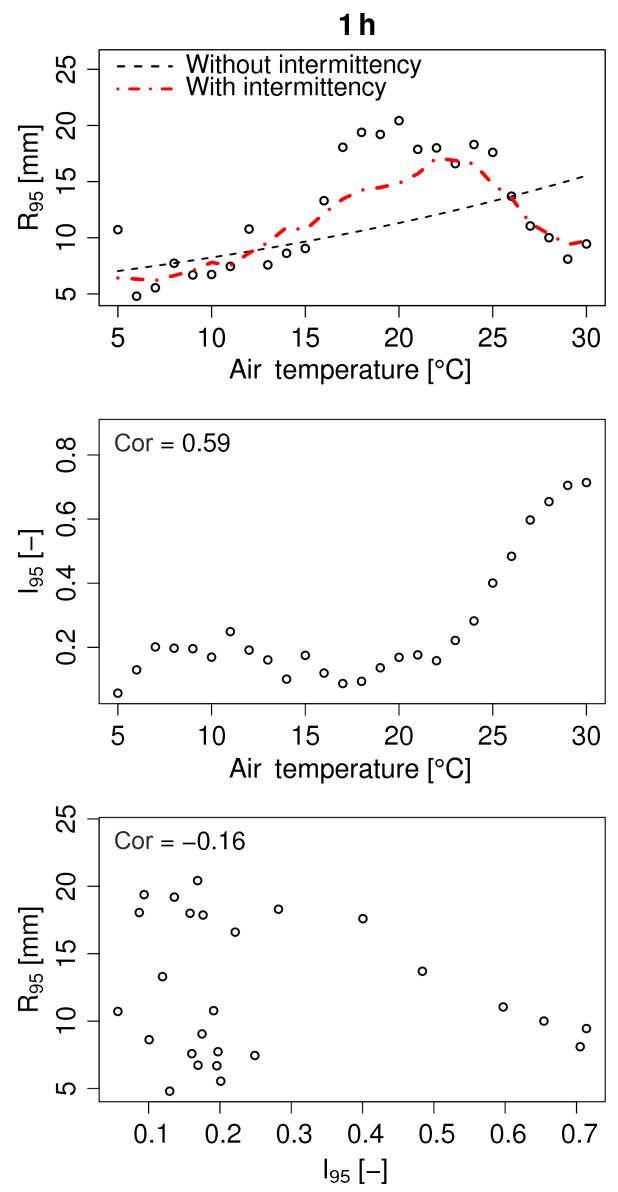

Figure 4. 95th quantile of rainfall amounts at the hourly timescale as a function of temperature and intermittency at AL-Fairhope-3NE. Black dots denote sample estimates. The black and red lines represent the fitted scaling models given in Eqs. (9) and (11).

$\frac{R_{q}(\Delta t, T)}{1-I_{q}(\Delta t, T)} \approx e^{\alpha_{q}^{\text {new }}(\Delta t)+\beta_{q}^{\text {new }}(\Delta t) T}$,
$\lambda_{q}^{\text {true }}(\Delta t)=e^{\beta_{q}^{\text {new }}(\Delta t)}-1$,

where $\lambda_{q}^{\text {true }}(\Delta t)$ represents the scaling rate with temperature for the intermittency corrected rainfall amounts. The goal of this paper is to study these scaling rates with temperature and intermittency across different regions and quantify their relative importance for a wide range of temporal aggregation scales.

\section{Results}

\subsection{The effect of intermittency on the scaling of rainfall amounts}

Figure 4 shows the 95th quantile of hourly rainfall accumulations at Fairhope as a function of air temperature, together with the corresponding intermittency estimates from 
the fitted generalized linear model in Eq. (5). The average rate of increase with temperature, as indicated by the black dotted line, is $3.2 \%{ }^{\circ} \mathrm{C}^{-1}$. However, there appears to be two distinct scaling patterns with temperature. The first, between 5 and $20^{\circ} \mathrm{C}$, is characterized by a steady increase in rainfall amounts of $6.5 \%{ }^{\circ} \mathrm{C}^{-1}$, in good agreement with the Clausius-Clapeyron relationship. The second part, from 20 to $30^{\circ} \mathrm{C}$, exhibits a negative trend in rainfall amounts of $-4.5 \%{ }^{\circ} \mathrm{C}^{-1}$ associated with a sudden and rapid increase in intermittency. This is consistent with previous evidence presented by Lenderink et al. (2011) and Berg et al. (2013), who pointed out similar changes in scaling above $22-23^{\circ} \mathrm{C}$. Lenderink et al. (2011) could not fully explain the reasons behind this but suggested it could be due to microphysical processes occurring in convective clouds. Berg et al. (2013) argue along a slightly different line. Their working hypothesis is that convective precipitation extremes scale much faster with temperature than stratiform extremes. The change in scaling rate at higher temperatures could therefore be explained by relative changes in the frequencies of stratiform over convective precipitation extremes beyond $22-$ $23^{\circ} \mathrm{C}$. Another explanation could be the existence of strong moisture limitations in the regions surrounding the rainfall and upwind thereof. The atmosphere might have capacity to hold more water at higher temperatures, but the land surfaces have no additional moisture to give, causing the relationship between temperature and rainfall extremes to change. The key parameter in this case is the rate at which new precipitable water can be evaporated and brought in from surrounding regions, which increases with temperature but will be limited by advection velocities and moisture availability at nearby land surfaces. A simple calculation of daily mean evaporation rates with temperature using the approximation provided by Linacre (1977) confirms this hypothesis, showing that although mean evaporation rates increase steadily with temperature, the rate of increases slows down at higher temperatures. Even in cases of unlimited moisture supply, evaporation rates remain small compared with precipitation rates. Thus, once all the water in a column of air has been rained out, the dominant factors controlling precipitation totals at scales beyond $1 \mathrm{~h}$ are likely to be dynamical in nature. Intermittency, although it is not a physical quantity, can be viewed as a summary statistic of the combined effect of all dynamical processes at work in rainfall. As such, it can help better understand the response of rainfall extremes to changing temperatures beyond simple Clausius-Clapeyron scaling. Indeed, looking at the top panel of Fig. 4, we see that the modified scaling model in Eq. (11) provides a much better description of the actual scaling pattern of the extremes. It correctly reproduces the observed decrease in rainfall quantiles at higher temperatures without the need to separate stratiform from convective events. Most importantly, it shows that once the change in intermittency has been accounted for, as detailed in Eq. (14), a clear and consistent positive trend of rainfall intensity of approximately $6.6 \%{ }^{\circ} \mathrm{C}^{-1}$ over the entire
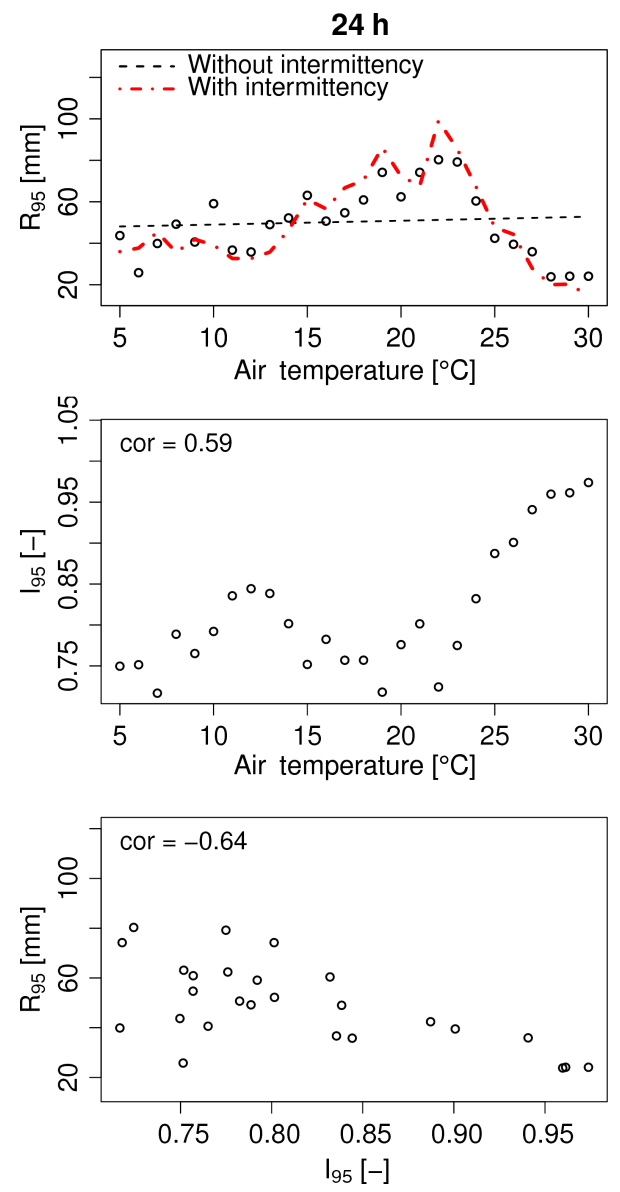

Figure 5. The 95th quantile of rainfall amounts at the daily timescale as a function of temperature and intermittency at ALFairhope-3-NE. Black dots denote sample estimates. The black and red lines represent the fitted scaling models given in Eqs. (9) and (11).

range of temperatures emerges. This $6.6 \%$ increase represents the true underlying thermodynamic trend with temperature of the water-holding capacity of air, on top of which an additional (non-linear) component due to intermittency has been added.

Figure 5 shows the same type of analysis for the station in Fairhope but this time at the daily aggregation scale. The top panel shows the effective scaling rate with temperature is close to zero, meaning that temperature alone is not a good predictor of rainfall accumulations at larger timescales. Intermittency, on the other hand, appears to exert a much stronger control over the total accumulation, as indicated by the strong rank correlation coefficient of -0.64 . Similarly to the hourly scale, there appears to be a sudden and rapid increase in internal intermittency at temperatures above $20^{\circ} \mathrm{C}$. The modified scaling model in Eq. (11) accounting for intermittency performs much better, predicting an increase in rainfall amounts of $5.6 \% \pm 1.4 \%$ per degree Celsius. This is slightly smaller than Clausius-Clapeyron scaling but still reasonable given 

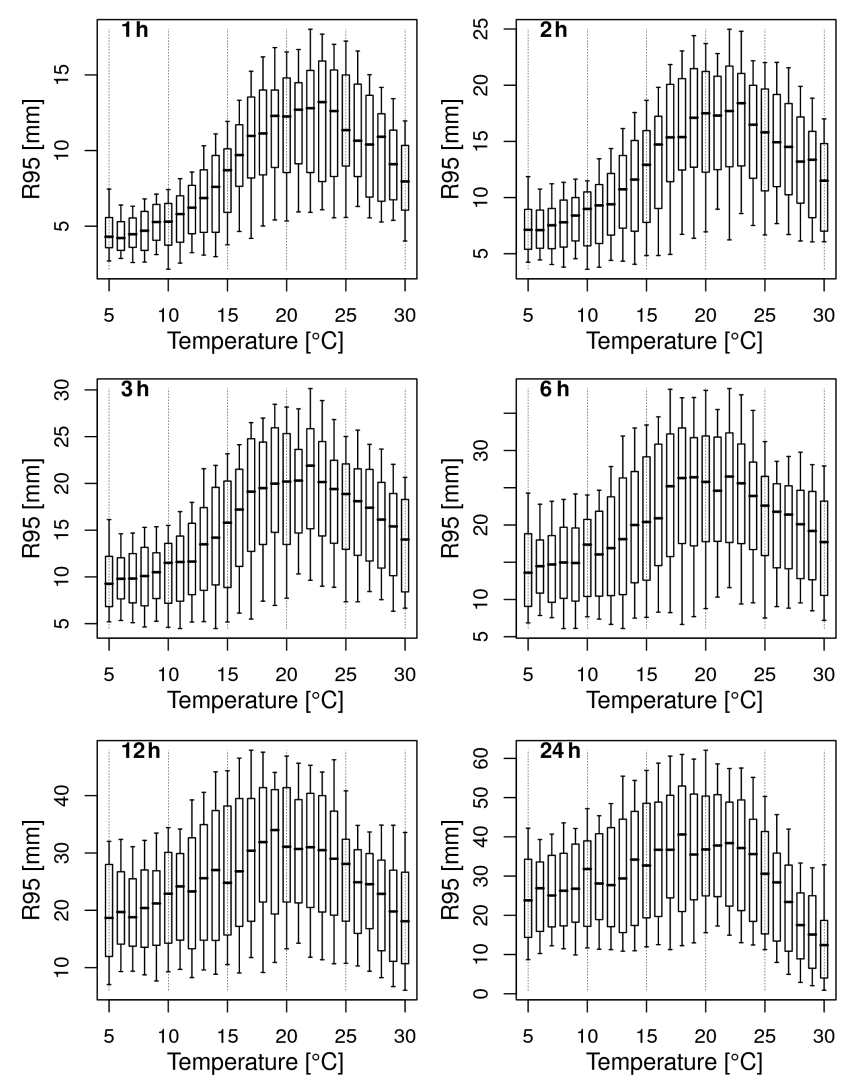

Figure 6. Box plots of 95 th rainfall quantile vs. temperature at 1,2 , $3,6,12$ and $24 \mathrm{~h}$ aggregation timescales.

the uncertainty and the fact that we only considered the 95th quantile of all rainfall amounts.

Similar analyses of the 95th rainfall quantiles and intermittency for all 99 stations in the dataset in Figs. 6 and 7 confirm this general scaling pattern. At the hourly scale and temperatures below $20^{\circ} \mathrm{C}$, the median intermittency of extremes tends to be very low. Temperature therefore naturally tends to play a much more important role in influencing rainfall amounts. However, as we move toward larger scales and higher temperatures, intermittency progressively gains in importance. The exact scale at which intermittency starts to exert more control over total amounts than temperature depends on the considered station. But overall, the transition usually occurs at temporal aggregation scales of 3 to $6 \mathrm{~h}$.

Figure 8 provides more insight into how intermittency affects the scaling of rainfall extremes with temperature and aggregation timescale. In the model without intermittency, scaling rates rapidly decrease with $\Delta t$ from approximately $4.37 \%$ at the hourly timescale to $-0.45 \%$ at the $24 \mathrm{~h}$ scale. The average uncertainty affecting the estimated scaling rates at a given timescale (among all stations) is between 1.8 and $2.7 \%$ and increases with $\Delta t$. The rapid decrease in scaling rate conveys the wrong idea that precipitation rates at larger scales do not depend on temperature. In reality, however, the
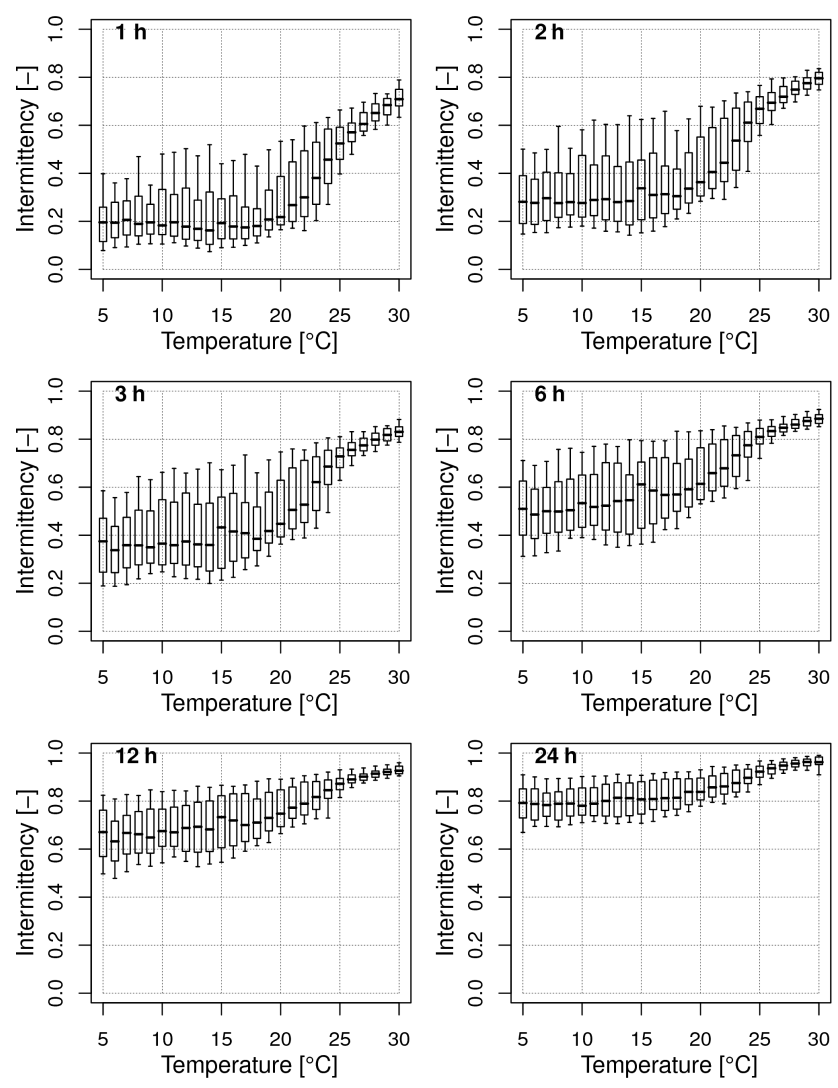

Figure 7. Box plots of internal intermittency vs. temperature for 1 , 2, 3, 6, 12 and $24 \mathrm{~h}$ aggregation timescales.
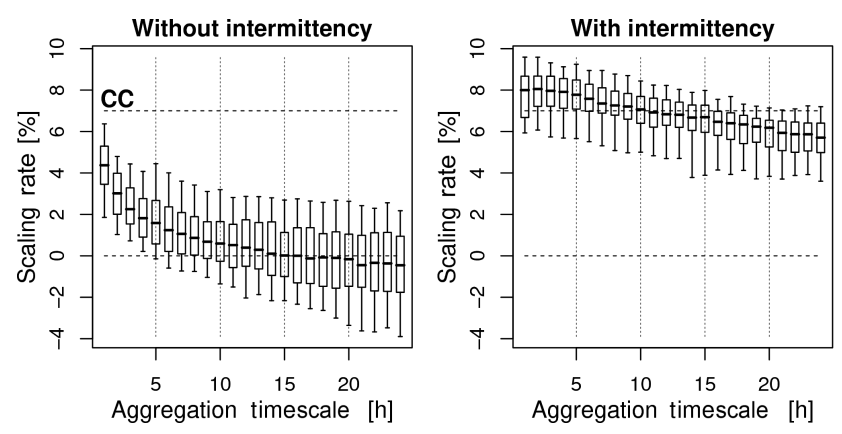

Figure 8. Box plots of estimated scaling rates of 95th rainfall quantile with temperature as a function of timescale. Each box plot shows the $0,25,50,75$ and $90 \%$ quantiles of all 99 stations in the dataset.

loss of scaling can be explained by a rapidly increasing intermittency at higher temperatures and aggregation timescales. In other words, intermittency acts as a confounding factor, masking the true scaling rate of rainfall rates with temperature. After correcting for it, the effect of temperature becomes visible again and results are much closer to what can be expected from the Clausius-Clapeyron relationship. Still, there appears to be a small decrease in the scaling rate with $\Delta t$ after correction for intermittency from $8.0 \%$ at the 
hourly scale to $5.70 \%$ at the $24 \mathrm{~h}$ scale. The latter however, can be explained by the relatively small sample sizes and is well within the range of uncertainty (average uncertainty of $1.3-1.7 \%$ per timescale, increasing with $\Delta t$ ).

The stations with the strongest scaling rates overall (both at the hourly and daily timescales) were FLSebring-23-SSE $(12.96 \% \pm 3.4 \%$ without intermittency and $14.70 \% \pm 1.74 \%$ with intermittency) and FL-EvergladesCity-5-NE $(12.42 \% \pm 5.15 \%$ and $13.04 \% \pm 2.41 \%)$, both situated in a humid tropical climate famous for large and intense warm season thunderstorms. Apart from these two, no other station exhibited scaling rates in excess of $12 \%{ }^{\circ} \mathrm{C}^{-1}$. In general, we observe that the lowest scaling rates with temperature (both corrected and uncorrected for intermittency) tend to be associated with moisture-limited places (e.g., CA-Stovepipe-Wells-1-SW, CA-Fallbrook-5NE, UT-Brigham-City-28-WNW and NM-Clayton-3-ENE). The state of California is a particularly interesting case. Uncorrected scaling rates at CA-Fallbrook-5-NE, for example, were $0.07 \% \pm 2.3 \%$ at the hourly timescale and $-10.42 \% \pm 3.13 \%$ at the daily timescale. The strong negative scaling rate at the daily timescale can be explained by the fact that, unlike the southern and central parts of the United States, large-scale precipitation extremes along the west coast usually occur during the cold season. They are associated with rapid transport of moisture from the Pacific Ocean towards the mainland along atmospheric rivers, which results in a very steady and persistent rain over time (Berg et al., 2002; Bracken et al., 2015; Lamjiri et al., 2017). The scaling model that corrects for changes in intermittency removes the negative trend with temperature. But even the corrected scaling rates remain relatively low at $1.67 \% \pm 1.85 \%$ for the hourly scale and $2.24 \% \pm 2.30 \%$ at the daily scale, confirming that large-scale moisture transport and storm dynamics play a much more important role than temperature in determining rainfall totals over this region of the globe.

Overall, the results confirm that air temperature alone is not systematically a good indicator for understanding extreme rainfall accumulations, and conditions in surrounding regions must be taken into account as well. The correction for intermittency makes it easier to understand and characterize the true sensitivity of heavy rainfall to changes in air temperatures across scales and geographical regions. But significant uncertainty remains, and the corrected model does not tell the full story either. However, it offers new insight into the nature of rainfall extremes, which is helpful in explaining some of the abnormally low/high scaling rates that we see in the observational record.

\subsection{Goodness of fit}

Repeating the same type of analysis as above, we computed the root mean square error (RMSE) and coefficient of determination $\left(R^{2}\right)$ of the two different scaling models (i.e., with/without intermittency) for all 99 stations in the dataset
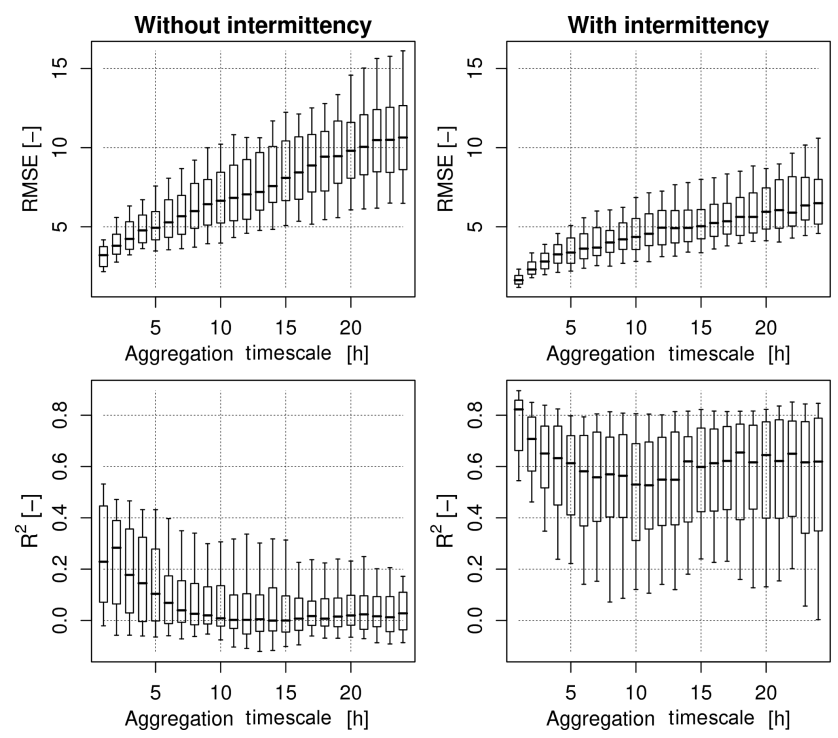

Figure 9. Box plots of root mean square error (RMSE) and coefficient of determination $\left(R^{2}\right)$ of the estimated 95th rainfall quantile as a function of scale. The model without intermittency is shown on the left and the model with intermittency on the right. Each box plot shows the 10, 25, 50, 75 and $90 \%$ quantiles of all 99 stations in the dataset.

and across all scales of aggregation (see Fig. 9). On average, the model that corrects for intermittency (on the right) reduced RMSE values by a factor 1.6 while increasing the coefficient of determination by 0.6 . It systematically outperformed the simpler model based on temperature alone and kept a reasonable goodness of fit across all aggregation timescales, especially at the larger ones where temperature alone performed poorly. The five biggest improvements in model performance at the hourly timescales were observed in the southern states at MS-Holly-Springs-4-N, AL-Russellville-4-SSE, LA-Lafayette-13-SE, LA-Monroe26-N and SC-McClellanville-7-NE, all characterized by large moisture availability and rapid increases in intermittency at higher temperatures. The five largest improvements at the $24 \mathrm{~h}$ timescale, on the other hand, were located in more moisture-limited places such as NM-Artesia-2-WNW, NMVaughn-36-SSE, TX-Port-Aransas-32-NNE, TX-Austin-33NW and UT-Blanding-26-SSW. In these regions, large storm totals strongly depend on intermittency and the steady transport of moisture from surrounding regions.

The comparisons above show that while temperature plays an important role in shaping rainfall extremes at smaller scales, its effects at larger scales are likely to be masked by changes in storm dynamics, such as increased intermittency. Additional correlation analyses between the 95th rainfall quantile and internal intermittency with temperature presented in Fig. 10 provide more insight into this phenomenon. They show that the median rank correlation between amounts and intermittency decreases from 0.12 at the hourly scale 


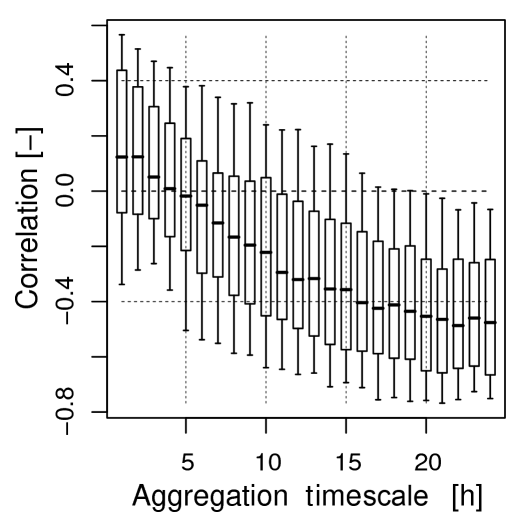

Figure 10. Box plots of Spearman rank correlation between the 95th rainfall quantile and intermittency (over temperature) as a function of timescale. Each box plot shows the 10, 25, 50, 75 and $90 \%$ quantiles of all 99 stations in the dataset.

to -0.48 at the daily scale. The fact that extremes at small aggregation scales below $3 \mathrm{~h}$ tend to be slightly positively correlated with intermittency is in agreement with the findings of Wasko et al. (2016). It means that small-scale rainfall extremes at higher temperatures tend to be more concentrated in space and time while rainfall extremes at scales above a few hours tend to be associated with longer-lasting systems like the passage of a cold front or a system of thunderstorms in which a series of convective cells repeatedly moves over the same region. The effect of temperature on total rainfall amounts in this case becomes less clear, as large accumulations can occur both at low and high temperatures. This interplay between temperature, peak intensity, intermittency and storm totals outlines a more complicated picture than traditionally depicted in Clausius-Clapeyron scaling analyses. It shows that rainfall extremes vary with temperature in ways that cannot be fully explained by Clausius-Clapeyron but require a more in-depth understanding of storm type, organization and dynamics. It also underlines why the ability to produce realistic storm dynamics and rainfall structures in global and regional climate models is so important for making credible projections about the future of rainfall extremes across scales.

\subsection{Sensitivity to choice of quantile}

So far, all results we have shown were for extremes exceeding the 95th quantile of rainfall accumulations. The goal of this last section is to quantify the sensitivity of the retrieved scaling rates with respect to the choice of the quantile $q$ used to identify the rainfall extremes in the first place. Previous studies have shown that relatively high quantiles are necessary in order to observe Clausius-Clapeyron scaling of rainfall extremes (Shaw et al., 2011). But a clear and detailed study of the influence of $q$ on observed scaling rates is still missing. The reason the choice of quantile is important for
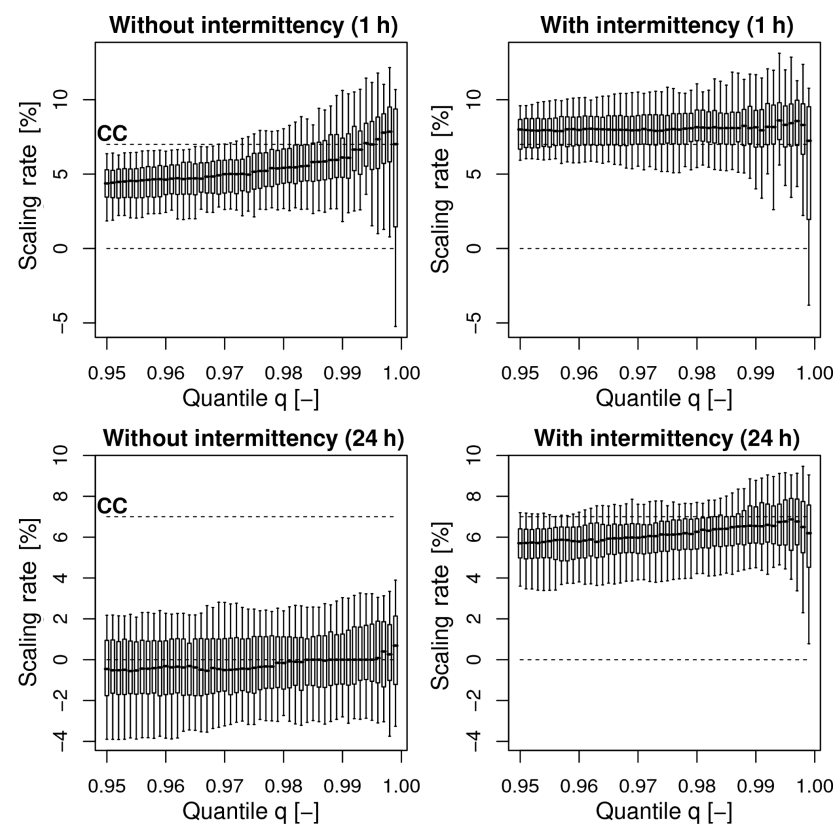

Figure 11. Box plots of rainfall scaling rates with temperature (5$30^{\circ} \mathrm{C}$ ) as a function of the quantile $q$ for the 1 and $24 \mathrm{~h}$ timescales. The model without correction for intermittency is shown on the left. The model with correction for intermittency is shown on the right. Each box plot shows the 10, 25, 5075 and $90 \%$ quantiles of all 99 stations in the dataset.

scaling analyses is that higher rainfall amounts naturally tend to be associated with lower intermittency levels. This makes them more likely to scale with temperature. However, since intermittency might not change uniformly with $q$ and $T$, changes in scaling might not be obvious to anticipate.

Figure 11 shows box plots of the estimated scaling rates of rainfall amounts with temperature at the 1 and $24 \mathrm{~h}$ aggregation timescales as a function of the considered quantile $q$. In the first model (without intermittency), scaling rates depend positively on the quantile $q$, increasing by more than $2 \%$ between the 95th quantile and the 99.5th quantile at the hourly timescale. By contrast, the model accounting for intermittency exhibits a much smaller sensitivity at the hourly scale. It consistently predicts a scaling rate of about $8 \%$ per degree Celsius, independently of the chosen quantile $q$. At the daily timescale, both models exhibit similar sensitivities to the choice of the quantile. However, the model without intermittency fails to capture the temperature dependence due to the strong influence of intermittency at this scale (see previous section). The model with intermittency predicts a more reasonable scaling rate of 5.7 to $6.9 \%$ per degree Celsius, slowly converging towards Clausius-Clapeyron scaling for larger values of $q$. The large spread for $q>0.995$ can be interpreted as a sign that the selected time series are not long enough to accurately estimate rainfall quantiles above the 99.5th quantile. 
Perhaps one of the most striking features in Fig. 11 is how sensitive the uncorrected scaling rates at the hourly timescale appear to be with respect to the choice of $q$. The shape of the trend could be used to support the idea that the largest rainfall extremes at small scales respond much faster to changes in temperature than expected from the Clausius-Clapeyron relationship. However, because the corrected scaling rates do not exhibit this trend, this increase with $q$ is likely to be a statistical artifact caused by intermittency.

To better understand this phenomenon, it is important to look at how quickly intermittency levels change when going from one temperature class to another and how this rate varies with $q$. Intuitively, the average intermittency of rainfall extremes tends to decrease with $q$, resulting in stronger overall sensitivity to temperature. However, the decrease in intermittency from one quantile to another may not necessarily be uniform across all temperature bins. Typically, extremes at higher temperatures, which are more intermittent, will see their intermittency decrease at a faster rate than extremes at lower temperatures. These nonuniform changes in intermittency between low and high temperatures can result in an apparent amplification of the scaling rate with temperature as we move towards larger $q$.

Figure 12 illustrates this scenario by showing the differences in observed rainfall amounts and intermittency for $q=0.95$ and $q=0.997$ vs. temperature for the station in Fairhope, Alabama. The average intermittency decreases from 0.262 for $q=0.95$ to 0.066 for $q=0.997$. However, this difference is not uniformly spread across all temperature bins. As shown in Fig. 12c, intermittency at higher temperatures tends to decrease faster than at lower temperatures. This results in an amplification of extremes at higher temperatures with $q$ beyond that expected by Clausius-Clapeyron. One might argue that, in the end, it does not really matter whether the increase in total rainfall amount is caused by larger peak intensity or decreasing intermittency, or a combination of both, as long as the net rate of change is known. However, looking beyond rainfall totals, one also needs to take into account the fact that hydrological response is a combination of rainfall amount and dynamics. Thus, the interplay between peak intensity, duration and total rainfall amounts with temperature across scales is a crucial factor to consider for flood risk analyses. In the end, storm water infrastructures need to be capable to deal with rainfall extremes across all relevant spatial and temporal scales. This requires more indepth knowledge of intermittency and rainfall dynamics as a function of atmospheric variables than is currently available.

\section{Conclusions}

Intermittency is a key feature controlling the variability in precipitation. Yet its effect is often poorly taken into account. The first main result of this study is that most rainfall extremes above hourly scales are intermittent in nature. For ex-
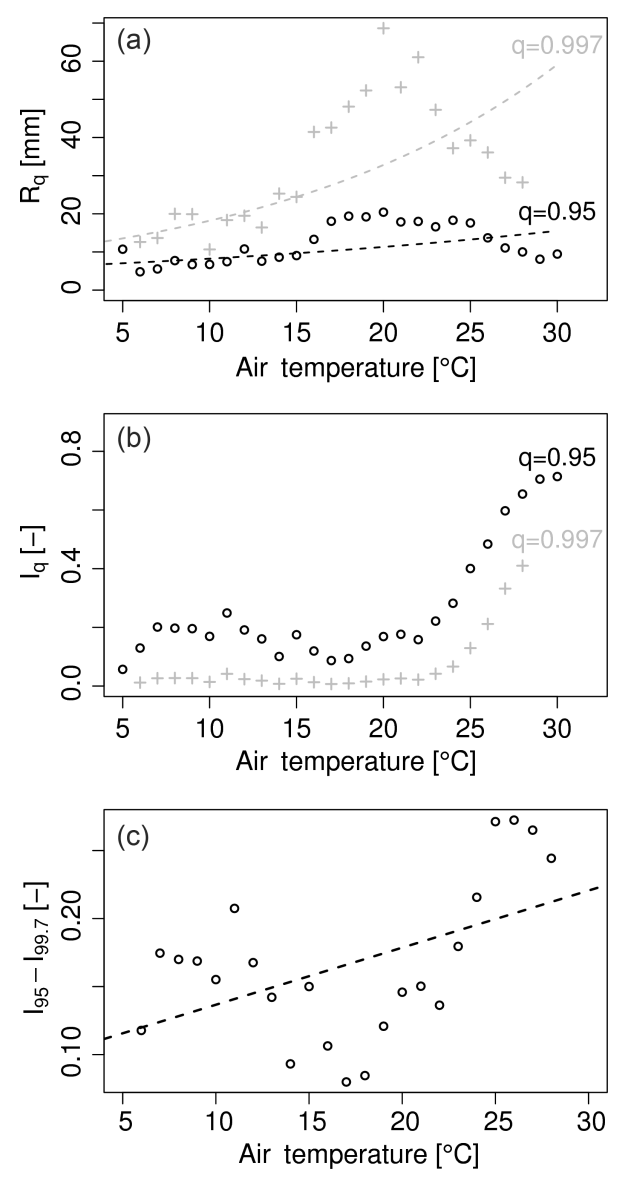

Figure 12. From (a) to (c): rainfall quantiles, internal intermittency and changes in intermittency at the hourly timescale as a function of temperature and two different quantiles $(q=0.95$ and $q=0.997)$ for the station AL-Fairhope-3-NE. The dashed line in (c) represents the fitted linear regression (using least squares) of $I_{95}-I_{99.7}$ as a function of air temperature.

ample, it is common for rainfall extremes at daily timescales to exhibit upward of $80 \%$ internal intermittency. For these reasons, peak intensity often turns out to be a rather weak predictor of total amounts compared with storm duration and dynamics.

The second important finding is that the current conceptual framework for studying the relationship between rainfall extremes and temperature based on Clausius-Clapeyron alone is too simplistic. Changes in extreme precipitation with temperature cannot be reduced to a single number. Instead, there appears to be a seamless progression of changes, starting at the sub-hourly scales where rainfall extremes are predominantly controlled by variations in temperature up towards hourly, daily and weekly extremes, which are increasingly dominated by storm dynamics and the organization of convection in larger weather systems. The combination of all these dynamical processes results in changing intermittency, which affects the sensitivity of extremes to temper- 
ature. Temperature itself remains a crucial factor across all timescales by controlling evaporation rates and the maximum moisture-holding capacity of the air. But because the rate at which new precipitable water can be brought in from surrounding regions is limited, net effective changes in rainfall totals with increasing temperatures are not necessarily well described by Clausius-Clapeyron scaling alone. New improved scaling models that take into account changes in intermittency, evaporation rates, advection speed and horizontal mass convergence can help to better separate the thermodynamic from the dynamic components, which leads to a more accurate depiction of precipitation extremes across scales. But the insight that these modified scaling laws provide is still limited, as rainfall-producing processes are complex and depend on many other physical factors like shifts in the horizontal and vertical circulations of the atmosphere, instabilities in the thermodynamic profile, aerosol concentrations and cloud microphysics.

Despite decades of development, current numerical weather prediction models and climate simulations still lack the ability to reproduce realistic intermittent rainfall patterns, especially at sub-daily timescales where convective processes are the most important contributors to extremes. As a result, projections about the future of rainfall extremes are still very uncertain. Perhaps, future developments might profit from the new scaling model proposed in this paper, allowing them to make a more in-depth analysis of how well precipitation extremes are simulated in numerical weather models and how realistically these vary with scale and temperature. New statistical metrics and diagnostic tools specifically designed to assess the realism of simulated intermittency patterns independently of total amounts and peak intensity might prove useful with regard to this issue (Schleiss and Smith, 2016). At the same time, more research is needed into the type of meteorological conditions capable of sustaining heavy rainfall intensities over a long period of time, including positive feedback mechanisms in mature storms, modifications of large-scale moisture transport by climate change and spatiotemporal organization of storms across scales, none of which are fully understood yet.

Finally, note that while the present work only focused on temporal intermittency, the same approach could be used to study the internal intermittency of extremes aggregated over different spatial scales, for example by looking at how the fraction of dry pixels within a fixed area responds to changes in temperature. Also, since intermittency and temperature are not sufficient to fully predict the response of heavy rainfall accumulations across scales, additional covariates like wind speed, dew point, pressure and vertical motion could be used in the analyses to further refine the models. Similarly, it might be worth looking at alternative intermittency metrics, like the fraction of the time the rainfall intensity exceeds a certain threshold or the temporal variability in the rainfall rate within an extreme. The latter might offer a more detailed picture of internal storm variability than the simple binary rain/no-rain approach used in this paper. A more detailed and systematic analysis of the joint probability distribution of $\left(R_{\Delta t}, T_{\Delta t}, I_{\Delta t}\right)$ and pairwise conditional density functions for all values of rainfall accumulations (and not only for the upper quantiles) might also be beneficial to better understand how rainfall amounts, temperature and intermittency are linked across scales.

Data availability. All data are freely accessible via anonymous ftp at ftp://ftp.ncdc.noaa.gov/pub/data/uscrn/products/subhourly01 (NOAA, 2018).

Competing interests. The authors declare that they have no conflict of interest.

Special issue statement. This article is part of the special issue "Hydro-climate dynamics, analytics and predictability". It is not associated with a conference.

Acknowledgements. The author would like to thank the National Oceanic and Atmospheric Administration (NOAA) for collecting and distributing the high-quality datasets used in this study.

Edited by: Naresh Devineni

Reviewed by: two anonymous referees

\section{References}

Alduchov, O. A. and Eskridge, R. E.: Improved Magnus Form Approximation of Saturation Vapor Pressure, J. Appl. Meteorol., 35, 601-609, https://doi.org/10.1175/15200450(1996)035<0601:IMFAOS>2.0.CO;2, 1996.

Allan, R. P. and Soden, B. J.: Atmospheric Warming and the Amplification of Precipitation Extremes, Science, 321, 1481-1484, https://doi.org/10.1126/science.1160787, 2008.

Azad, R. and Sorteberg, A.: Extreme daily precipitation in coastal western Norway and the link to atmospheric rivers, J. Geophys. Res.-Atmos., 122, 2080-2095, https://doi.org/10.1002/2016JD025615, 2017.

Ban, N., Schmidli, J., and Schär, C.: Heavy precipitation in a changing climate: Does short-term summer precipitation increase faster?, Geophys. Res. Lett., 42, 1165-1172, https://doi.org/10.1002/2014GL062588, 2015.

Barbero, R., Fowler, H. J., Lenderink, G., and Blenkinsop, S.: Is the intensification of precipitation extremes with global warming better detected at hourly than daily resolutions?, Geophys. Res. Lett., 44, 974-983, https://doi.org/10.1002/2016GL071917, 2017.

Berg, P., Moseley, C., and Haerter, J. O.: Strong increase in convective precipitation in response to higher temperatures, Nat. Geosci., 6, 181-185, https://doi.org/10.1038/ngeo1731, 2013. 
Berg, W., Kummerow, C., and Morales, C. A.: Differences between East and West Pacific rainfall systems, J. Climate, 15, 3659-3672, https://doi.org/10.1175/15200442(2002)015<3659:DBEAWP>2.0.CO;2, 2002.

Bracken, C., Rajagopalan, B., Gangopadhyay, S., and Alexander, M.: Spatial Variability of Seasonal Extreme Precipitation in the Western United States, J. Geophys. Res.-Atmos., 120, 45224533, https://doi.org/10.1002/2015JD023205, 2015.

De Michele, C. and Ignaccolo, M.: New perspectives on rainfall from a discrete view, Hydrol. Process., 27, 2379-2382, https://doi.org/10.1002/hyp.9782, 2013.

Diamond, H. J., Karl, T. R., Palecki, M. A., Baker, C. B., Bell, J. E., Leeper, R. D., Easterling, D. R., Lawrimore, J. H., Meyers, T. P., Helfert, M. R., Goodge, G., and Thorne, P. W.: U.S. Climate Reference Network after One Decade of Operations: Status and Assessment, B. Am. Meteorol. Soc., 94, 485498, https://doi.org/10.1175/BAMS-D-12-00170.1, 2013.

Donat, M. G., Alexander, L. V., Herold, N., and Dittus, A. J.: Temperature and precipitation extremes in centurylong gridded observations, reanalyses, and atmospheric model simulations, J. Geophys. Res.-Atmos., 121, 11174-11189, https://doi.org/10.1002/2016JD025480, 2016.

Drobinski, P., Alonzo, B., Bastin, S., Silva, N. D., and Muller, C.: Scaling of precipitation extremes with temperature in the French Mediterranean region: what explains the hook shape?, J. Geophys. Res.-Atmos., 121, 3100-3119, https://doi.org/10.1002/2015JD023497, 2016.

Dunkerley, D.: Intra-event intermittency of rainfall: an analysis of the metrics of rain and no-rain periods, Hydrol. Process., 29, 3294-3305, 2015.

Dwyer, J. G. and O'Gorman, P. A.: Changing duration and spatial extent of midlatitude precipitation extremes across different climates, Geophys. Res. Lett., 44, 5863-5871, https://doi.org/10.1002/2017GL072855, 2017.

Frei, C., Scholl, R., Fukutome, S., Schmidli, R., and Vidale, P. L.: Future change of precipitation extremes in Europe: Intercomparison of scenarios from regional climate models, J. Geophys. Res., 111, B06105, https://doi.org/10.1029/2005JD005965, 2006.

Groisman, P. Y., Knight, R. W., Easterling, D. R., Karl, T. R., Hegerl, G. C., and Razuvaev, V. N.: Trends in Intense Precipitation in the Climate Record, J. Climate, 18, 1326-1350, https://doi.org/10.1175/JCLI3339.1, 2015.

Haerter, J. O. and Berg, P.: Unexpected rise in extreme precipitation caused by a shift in rain type?, Nat. Geosci., 2, 372-373, https://doi.org/10.1038/ngeo523, 2009.

Haerter, J. O., Berg, P., and Hagemann, S.: Heavy rain intensity distributions on varying time scales and at different temperatures, J. Geophys. Res.-Atmos., 115, D17102, https://doi.org/10.1029/2009JD013384, 2010.

Ignaccolo, M., De Michele, C., and Bianco, S.: The Droplike Nature of Rain and Its Invariant Statistical Properties, J. Hydrometeorol., 10, 79-95, https://doi.org/10.1175/2008JHM975.1, 2009.

Ivancic, T. J. and Shaw, S. B.: A U.S. based analysis of the ability of the Clausius-Clapeyron relationship to explain changes in extreme rainfall with changing temperature, J. Geophys. Res.-Atmos., 121, 3066-3078, https://doi.org/10.1002/2015JD024288, 2016.

Jeannin, N., Féral, L., Sauvageot, H., Castanet, L., and Lemorton, J.: Statistical distribution of the fractional area affected by rain, J. Geophys. Res., 113, D21120, https://doi.org/10.1029/2008JD009780, 2008.

Kumar, P. and Foufoula-Georgiou, E.: Characterizing Multiscale Variability of Zero Intermittency in Spatial Rainfall, J. Appl. Meteorol., 33, 1516-1525, https://doi.org/10.1175/15200450(1994)033<1516:CMVOZI>2.0.CO;2, 1994.

Kundu, P. K. and Siddani, R. K.: Scale dependence of spatiotemporal intermittency of rain, Water Resour. Res., 47, W08522, https://doi.org/10.1029/2010WR010070, 2011.

Lamjiri, M. A., Dettinger, M. D., Ralph, F. M., and Guan, B.: Hourly Storm Characteristics along the U.S. West Coast: Role of Atmospheric Rivers in Extreme Precipitation, Geophys. Res. Lett., 44, 7020-7028, https://doi.org/10.1002/2017GL074193, 2017.

Lenderink, G. and van Meijgaard, E.: Increase in hourly precipitation extremes beyond expectations from temperature changes, Nat. Geosci., 1, 511-514, https://doi.org/10.1038/ngeo262, 2008.

Lenderink, G., Mok, H. Y., Lee, T. C., and van Oldenborgh, G. J.: Scaling and trends of hourly precipitation extremes in two different climate zones - Hong Kong and the Netherlands, Hydrol. Earth Syst. Sci., 15, 3033-3041, https://doi.org/10.5194/hess-153033-2011, 2011.

Lepore, C., Veneziano, D., and Molini, A.: Temperature and CAPE dependence of rainfall extremes in the eastern United States, Geophys. Res. Lett., 42, 74-83, https://doi.org/10.1002/2014GL062247, 2015.

Linacre, E. T.: A simple formula for estimating evaporation rates in various climates, using temperature data alone, Agr. Meteorol., 18, 409-424, https://doi.org/10.1016/0002-1571(77)900073, 1977.

Mascaro, G., Deidda, R., and Hellies, M.: On the nature of rainfall intermittency as revealed by different metrics and sampling approaches, Hydrol. Earth Syst. Sci., 17, 355-369, https://doi.org/10.5194/hess-17-355-2013, 2013.

Muschinski, T. and Katz, J. I.: Trends in hourly rainfall statistics in the United States under a warming climate, Nat. Clim. Change, 3, 577-580, https://doi.org/10.1038/nclimate1828, 2013.

Neelin, J. D., Sahany, S., Stechmann, S. N., and Bernstein, D. N.: Global warming precipitation accumulation increases above the current-climate cutoff scale, P. Natl. Acad. Sci. USA, 114, 12581263, https://doi.org/10.1073/pnas.1615333114, 2017.

NOAA - National Oceanic and Atmospheric Administration: Quality controlled datasets, subhourly, https://www1.ncdc.noaa.gov/ pub/data/uscrn/products/subhourly01/, last access: 6 July 2018.

Panthou, G., Mailhot, A., Laurence, E., and Talbot, G.: Relationship between Surface Temperature and Extreme Rainfalls: A MultiTime-Scale and Event-Based Analysis, J. Hydrometeorol., 15, 1999-2011, https://doi.org/10.1175/JHM-D-14-0020.1, 2014.

R Core Team: R: A Language and Environment for Statistical Computing, R Foundation for Statistical Computing, Vienna, Austria, http://www.R-project.org/ (last access: 6 July 2018), 2017.

Scherrer, S. C., Fischer, E. M., Posselt, R., Liniger, M. A., Croci-Maspoli, M., and Knutti, R.: Emerging trends in heavy precipitation and hot temperature extremes in Switzerland, J. Geophys. Res.-Atmos., 121, 2626-2637, https://doi.org/10.1002/2015JD024634, 2016.

Schleiss, M. and Smith, J. A.: Two simple metrics for quantifying rainfall intermittency: the burstiness and mem- 
ory of inter-amount times, J. Hydrometeorol., 17, 421-436, https://doi.org/10.1175/JHM-D-15-0078.1, 2016.

Schleiss, M., Jaffrain, J., and Berne, A.: Statistical analysis of rainfall intermittency at small spatial and temporal scales, Geophys. Res. Lett., 38, L18403, https://doi.org/10.1029/2011GL049000, 2011.

Sen, P. K.: Estimates of the regression coefficient based on Kendall's tau, J. Am. Stat. Assoc., 63, 1379-1389, https://doi.org/10.1080/01621459.1968.10480934, 1968.

Shaw, S. B., Royem, A. A., and Riha, S. J.: The Relationship between Extreme Hourly Precipitation and Surface Temperature in Different Hydroclimatic Regions of the United States, J. Hydrometeorol., 12, 319-325, https://doi.org/10.1175/2011JHM1364.1, 2011.

Trenberth, K. E.: Changes in precipitation with climate change, Clim. Res., 47, 123-138, https://doi.org/10.3354/cr00953, 2011.
Trenberth, K. E., Dai, A., Rasmussen, R. M., and Parsons, D. B.: The Changing Character of Precipitation, B. Am. Meteorol. Soc., 84, 1205-1217, https://doi.org/10.1175/BAMS-84-9-1205, 2003.

Wasko, C., Sharma, A., and Johnson, F.: Does storm duration modulate the extreme precipitation-temperature scaling relationship?, Geophys. Res. Lett., 42, 8783-8790, https://doi.org/10.1002/2015GL066274, 2015.

Wasko, C., Sharma, A., and Westra, S.: Reduced spatial extent of extreme storms at higher temperatures, Geophys. Res. Lett., 43, 4026-4032, https://doi.org/10.1002/2016GL068509, 2016.

Westra, S., Fowler, H. J., Evans, J. P., Alexander, L. V., Berg, P., Johnson, F., Kendon, E. J., Lenderink, G., and Roberts, N. M.: Future changes to the intensity and frequency of short-duration extreme rainfall, Rev. Geophys., 52, 522-555, 2014. 